\title{
Converged Architecture for Broadcast and Multicast Services in Heterogeneous Network
}

\author{
Deokhui Lee*, Woosuk Kim ${ }^{\dagger}$, Byungjun Bae ${ }^{\dagger}$, Hyoungsoo $\mathrm{Lim}^{\dagger}$, and Jaewoo So* \\ ${ }^{*}$ Department of Electronic Engineering, Sogang University, Republic of Korea \\ ${ }^{\dagger}$ Broadcasting System Research Department, Electronics and Telecommunications Research Institute Daejeon, Republic of Korea \\ *jwso@sogang.ac.kr
}

\begin{abstract}
This paper proposes a converged architecture for broadcast and multicast services in a heterogeneous network. To support the broadcast/multicast services in the long-term evolution (LTE) network, the standard enhances multimedia broadcast multicast service (MBMS). In the broadcast network, digital video broadcasting for handhelds (DVB-H) is the standard for broadcasting IP data services to user equipment (UE). Accordingly, the converged LTE and DVB-H network can be used to service the broadcast/multicast services. To employ the converged network, additional entities are required for the contents management, electronic service guide (ESG) management and resource management. Therefore, we propose several logical entities to fulfill functionality from the above mentioned issues. The proposed logical entities are as follows: an integrated contents server, an integrated ESG server and an integrated management server. We also present several scenarios for implementing the converged architecture and compare the advantage of each scenario. Service providers can exploit the converged architecture according to preference and equipped devices. This study is of particular interest to the service providers because it provides the service providers with an insight of the various scenarios to make the converged architecture for broadcast/multicast service.
\end{abstract}

Index Terms-Broadcasting, converged architecture, digital video broadcasting for handhelds (DVB-H), multimedia broadcast multicast service (MBMS), long-term evolution (LTE).

\section{INTRODUCTION}

By increasing the traffic load of multimedia contents in cellular networks, such as mobile TV and sports broadcasting, it is important that a mobile operator efficiently provides multimedia contents. To cope with this issue, the 3rd generation partnership project (3GPP) standard defines the multimedia broadcast multicast services (MBMS) to increase the efficiency in the transmission of the same contents to several users. MBMS provides point-to-multipoint ( $\mathrm{p}-\mathrm{t}-\mathrm{m}$ ) radio bearers and multicast support in the cellular network to support the streaming and file download services. The above mentioned operation of MBMS is defined when MBMS over a single frequency network (MBSFN) is released [1]. Recently, long term evolution (LTE) standard enhances MBMS which is called enhanced-MBMS (E-MBMS) to provide more efficient delivery of broadcast/multicast. To improve the efficiency of MBMS, LTE includes old feature of MBMS (e.g. the use of $\mathrm{SFN}$ ) and the use of extended cyclic prefix (CP) to support large MBSFN networks [2].
Digital video broadcasting for handhelds (DVB-H) is a digital broadcast standard for IP data services, called IP datacast [3]. A DVB-H network is a unidirectional broadcasting network to provide contents to the many users. DVB-H will transmit the same data to as many uses as possible in extensive coverage area of DVB-H networks compared with that of cellular networks.

In the previous networks, the LTE and DVB-H networks are operated separately. To increase an efficiency of broadcast/multicast services, however, LTE and DVB-H networks will be integrated as the converged network. When mobile operator operates separately each network, several problems exist as follows: In the DVB-H network, the radio resource will be wasted if only a few users request the same content. And also, LTE network can serve restricted users because the available radio resource is limited. Therefore, the approach about the converged network is needed to exploit a complementary management.

Many researchers have endeavored to develop the converged architecture or platform to simultaneously employ the heterogenous networks [4]-[6]. In [4], the converged broadcast and telecommunication service platform is presented that is able to create, deliver, and manage content and services. The author of [5] referred that a subsystem insuring system control and signaling between broadcast and mobile networks is necessary. And also, the author mentioned that the most important tasks of the subsystem are the management and optimization of the available resources. In [6], the playout server and the service management server are proposed for converged DVB-H and UMT network architecture. The role of two servers is similar to that of subsystem in [5]. The playout server creates the timesliced streams or IP streams. The service management sever allocates radio resources, and manages the intersystem handover between DVB-H and UMTS. In [7], the proposed hybrid architecture provides a hybrid conditional access system among various communication networks such as advanced terrestrial digital multimedia broadcasting (ATDMB) and communication networks.

However, although [4]-[7] studied various architectures to manage the converged network, the conventional researches don't consider MBMS of cellular network. In other words, the conventional studies consider a convergence between unicast of the cellular networks and broadcast/multicast of the 


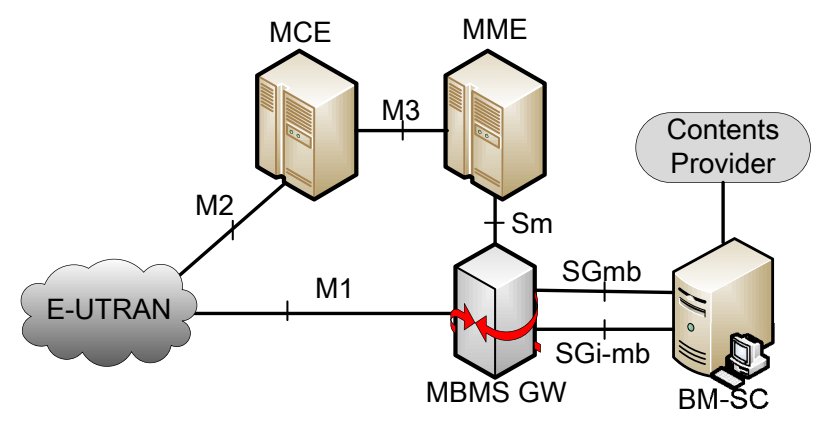

Fig. 1. E-MBMS logical architecture

broadcast networks. In this paper, we develop the converged network for broadcast/multicast services between LTE and DVB-H networks.

The remaining part of this paper is organized as follows: Section II contains the description of each network overview. In Section III, we propose logical entities for converged network. We provide several scenarios for converged architecture in Section IV and finally, Section V presents conclusions.

\section{NETWORK OVERVIEW}

\section{A. User Equipment}

A user equipment (UE) has to receive the signal from LTE or DVB-H network. There are two alternatives that either the UE has two independent receivers, or the UE has a single dual-mode receiver [6]. We assume that the UE has a single dual-mode receiver because it will benefit the UE in aspects of complexity and cost.

\section{B. Evolved Multimedia Broadcast Multicast Service (E- $M B M S)$}

Figure 1 shows the architecture of an E-MBMS system. The broadcast/multicast-service center (BM-SC) provides and delivers the cellular broadcast services. The BM-SC is located between the contents provider and the core network. It is responsible for contents delivery services that use MBMS. In addition, BM-SC provides service announcements for UEs. The service announcements contain essential information such as IP multicast addresses, multicast service identifier, time of transmission and media description. The BM-SC also informs the procedure of session start/stop for E-MBMS transmissions, and manages security and billing tasks function.

The 3GPP defines the MBMS gateway (MBMS GW) that is presented between BM-SC and eNBs whose principal functions is the sending/broadcasting of MBMS packets to each eNB transmitting the service. The MBMS GW uses IP Multicast as the means of forwarding MBMS user data to the eNB. The MBMS GW also can communicate with multiple control plane entities. It performs MBMS session control signaling (Session start/stop) towards the evolved universal terrestrial radio access network (E-UTRAN) via mobility management entity (MME) to set up MBMS radio bearers.

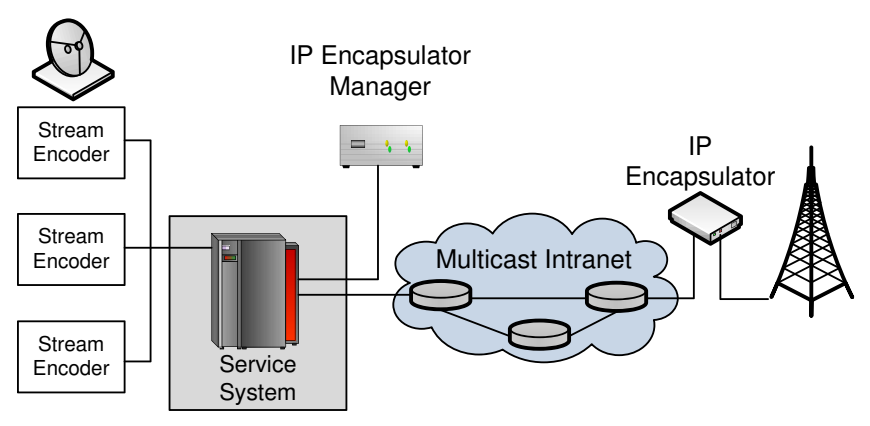

Fig. 2. DVB-H architecture

The multi-cell/multicast coordination entity (MCE) is a logical entity to manage the admission control and the allocation of the radio resources used by all the eNodeBs in the MBSFN area for multi-cell MBMS transmission using MBSFN operation. The radio resource allocation includes the modulation and coding scheme. The MCE is involved in MBMS session control signaling. However, it does not perform UE - MCE signaling.

\section{DVB-H Network}

The system architecture of a DVB-H system is presented in Fig. 2. The IP datacast service is used in the DVB-H system. Therefore, the DVB-H architecture shows various components and elements for IP datacast (IDPC).

The service system component is used to produce the various IP streams to the network. The IP streams are distributed over the multicast intranet to the IP encapsulator. The IP encasultor will produce the DVB-H transport stream (TS) with time slicing and multiprotocol encapsulation-forward error correction (MPE-FEC) included. Then, the received DVB-H TS from the IP encapsulator will be transmitted to the UE from the DVB-H transmitter.

\section{Logical Entities of Converged Architecture}

To integrate LTE with DVB-H network, we propose several logical entities. It consists of the integrated contents server, ESG server, management server as shown in Fig. 3. The functions of each logical entity can be subdivided into several logical entities and be integrated into any logical entity by any original equipment manufacturer (OEM). The proposed logical entities are described as follows:

\section{A. Integrated Contents Sever}

The integrated contents sever is responsible for providing encoded contents for each network according to the types of network and UE. It provides functions such as processing of MPEG encoder, transraters and transcoders (MPEG-2 to MPEG-4). The encoder is required to encode audio and video from contents provider. And also, the UE's graphical user interface (GUI) is created by integrated contents server. To represent the image of GUI, extensible markup language (XML), joint photographic experts group (JPEG) and portable network graphics (PNG) can be used. 


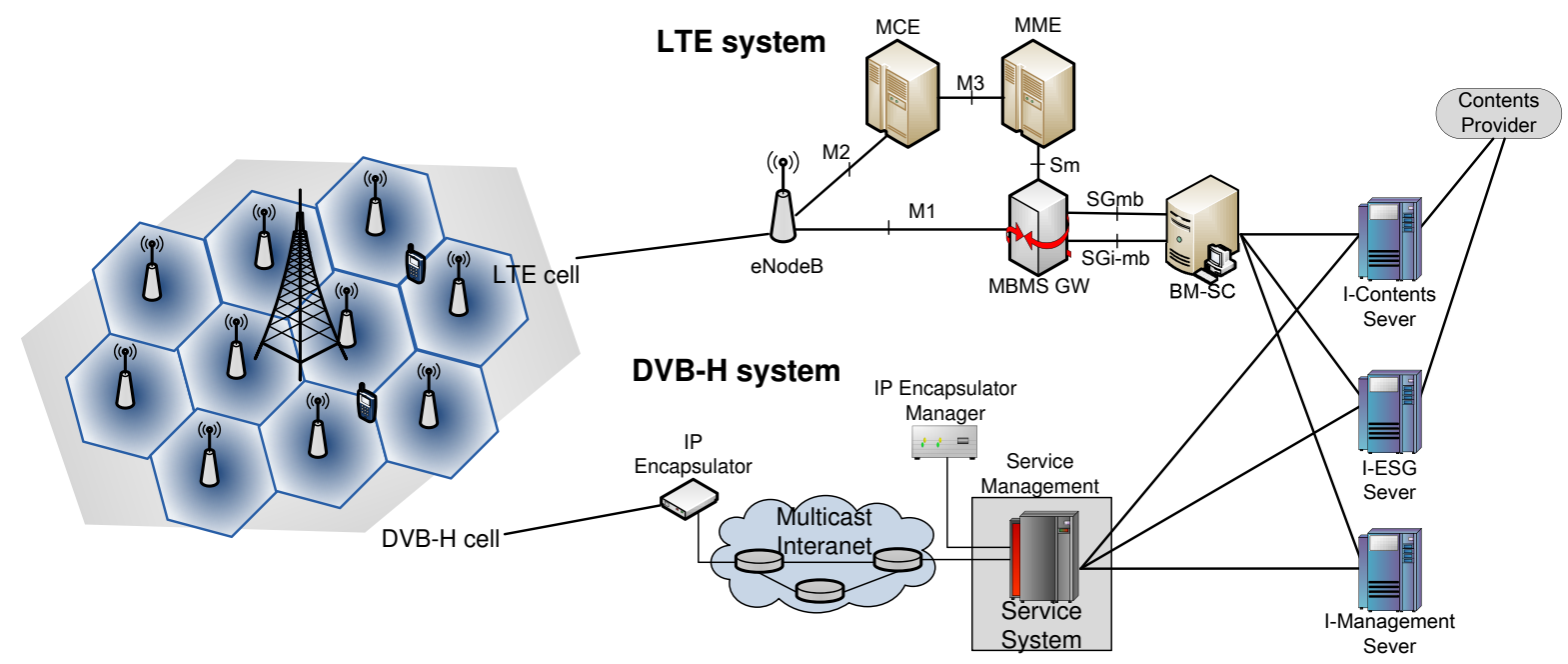

Fig. 3. Scenario 1: The architecture for converged network between LTE and DVB-H

\section{B. Integrated ESG server}

The purpose of ESG is to provide description of broadcast programming or scheduling information for current and upcoming programming. The ESG is dynamically updated and provided to users who use various broadcast/multicast services. If the ESG is created by LTE and DVB-H network respectively, it increases the complexity of overall networks and brings a burden in aspects of hardware. Therefore, it is important that the ESG for LTE and DVB-H network is simultaneously created and delivered by the integrated ESG server.

\section{Integrated Management Server}

A role of the integrated management sever is to allocate the radio resource and to decide the handover between LTE and DVB-H network. The integrated management server determines which channel should be used from the network to deliver contents. For example, the number of users who request the broadcast/multicast service can be considered as criteria for radio resource allocation.

Because the cell coverage of the DVB-H network is usually larger than that of the LTE network, the several cells of LTE network are overlapped by one DVB-H cell. Therefore, the scenario for handover between LTE and DVB-H network can be divided into three scenarios [6]: 1) handover within the DVB-H network; 2) handover within the LTE network; and 3) handover between the LTE and DVB-H network. In this paper, we assume that the integrated management sever handles the handover between the LTE and DVB-H network. The handover criteria is not only physical layer constraints such as received signal-to-noise ratio (SNR) of UE but also cost aspects like capital expenditure (CAPEX) and operating expenses (OPEX). It is important that the service provider considers the economic issues to exploit the converged architecture for broadcast/multicast services.

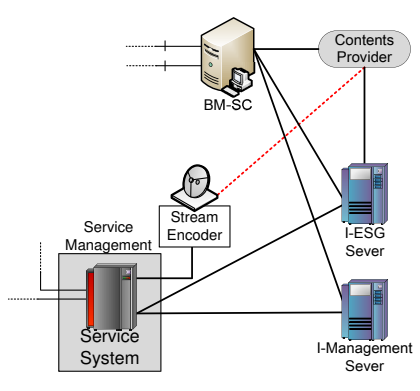

(a)

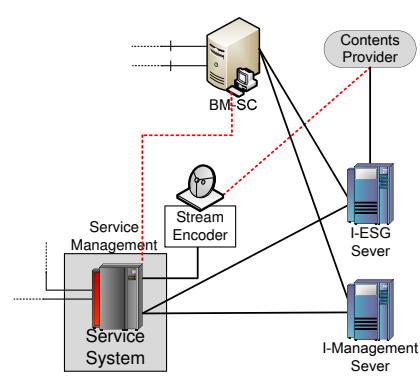

(b)
Fig. 4. The architecture for converged network between LTE and DVB-H, (a) Scenario 2 (b) Scenario 3

\section{Proposed Converged Architecture}

We propose three scenarios to design the converged architecture between LTE and DVB-H network. Each scenario has different component according to the utilization method of the logical entities.

For scenario 1, the architecture for the converged network is illustrated in Fig. 3. The contents provider is connected by the integrated contents server. It delivers the encoded contents to both BM-SC of LTE network and service system of DVB-H network. Because the procedure for encoding is processed in advance at the integrated contents sever, the stream encoder of the DVB-H network is not used in the case of scenario 1. And also, each integrated ESG and management server is connected by BM-SC of LTE network and service system of DVB-H network.

For scenario 2 and 3, the overall architecture is similar to that of scenario 1 except the part of contents processing, and the integrated contents server is not necessary. The architecture for scenario 2 and 3 is illustrated in Fig. 4. For the scenario 2, the contents provider is connected by BM-SC of LTE network and stream encoder of DVB-H network. Each network encodes independently the contents. The LTE and DVB-H network 
encode the contents using the stream encoder and the BM$\mathrm{SC}$, respectively. Actually, the standards do not mention about the function of encoding in BM-SC. However, the function of encoding can be added by manufacturers. For the scenario 3 , the contents provider is connected by a stream encoder of the DVB-H network, and not by BM-SC of the LTE network. To provide encoded contents for the LTE network, the service system of the DVB-H is connected to the BM-SC. In case of scenario 3, the BM-SC doesn't encode the contents data because BM-SC receives the encoded contents from DVB-H network.

\section{CONCLUSION}

A converged architecture was proposed for an integrated network between LTE and DVB-H network. To exploit the converged network, we propose several scenarios. In the future, the converged network between communication and broadcasting network will be operated to increase an efficiency of radio resource and service quality. Therefore, this is important to develop a converged architecture for broadcast/multicast services between communication and broadcast network because each network is operated independently. Several scenarios for converged network can give diversity to service providers because they can exploit the converged network according to their preference or equipped devices. This work can be developed and changed by considering the other issues such as cost, utilization metric and so on.

\section{ACKNOWLEDGMENT}

This research was funded by the MSIP(Ministry of Science, ICT \& Future Planning), Korea in the ICT R\&D Program 2013.

\section{REFERENCES}

[1] 3GPP: TS 25.346, "Introduction of the multimedia broadcast/multicast service (MBMS) in the radio access network (RAN)-Stage 2," Mar. 2008 , Version 7.7.0

[2] J. Monserrat, J. Calabuig, A. F. Aguilella, and D. G. Barquero, "Joint delivery of unicast and E-MBMS services in LTE networks," IEEE Trans. Broadcasting, vol. 58, no. 2, pp. 157-167, Jun. 2012.

[3] ETSI EN 302 304, "Digital video broadcasting (DVB); transmission system for handheld terminals (DVB-H)," Nov. 2004, Version 1.1.1.

[4] F. Allamandri and et. al, "Service platform for converged interactive broadband broadcast and cellular wireless," IEEE Trans. Broadcasting, vol. 53, no. 1, pp. 200-212, Mar. 2007.

[5] C. Heuck, "An analytical approach for performance evaluation of hybrid (broadcast/mobile) networks," IEEE Trans. Broadcasting, vol. 56, no. 1, pp. 9-18, Mar. 2010.

[6] X. Yang and T. Owens, "Intersystem soft handover for converged DVBH and UMTS networks," IEEE Trans. Veh. Technol., vol. 57, no. 3, pp. 1887-1898, May 2008.

[7] B. Bae, Y.-J. Song, S.-I. Lee, H.-Y. Seo, and J.-D. Kim, "An efficient and flexible hybrid conditional access system for advanced T-DMB," ETRI Journal, vol. 33, no. 4, pp. 629-632, 2011.

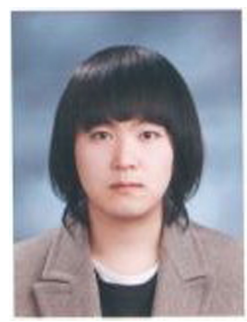

Deokhui Lee received the B.S. and M.S. degrees in electronic engineering from Sogang University, Seoul, Korea, in 2010 and 2012, respectively. He has been Ph.D. candidate from Sogang University since 2012. His research interests include resource management and performance analysis of various communication systems such as relay networks and MU-MIMO systems.

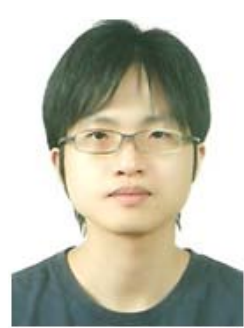

Woosuk Kim received the B.S. and M.S. in electrical \& computer engineering in 2001 and 2003 respectively from Hanyang University, Korea. And he received the Ph.D. in systems \& information engineering in 2012 from Tsukuba University, Japan. Since 2003, he has been with Electronics and Telecommunication Research Institute (ETRI), Korea. His major research interests include digital mobile broadcasting and human-computer interfaces.

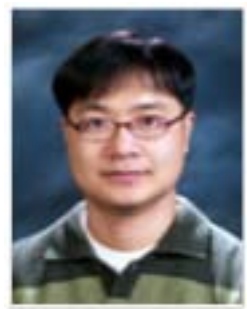

Byungjun Bae received the B.S., M.S., and Ph.D. degrees in electronics engineering from Kyungpook National University, Korea in 1995, 1997, and 2006 , respectively. From 1997 to 2000, he was a researcher at LG Electronics Inc. where he works on digital signal processing in digital television. Since 2000 , he has been with the broadcasting system research department in Electronics and Telecommunications Research Institute (ETRI) in Daejeon, Korea. His current research interests include the digital signal processing, digital broadcasting protocol processing, digital multimedia broadcasting system, and hybrid broadcasting services.

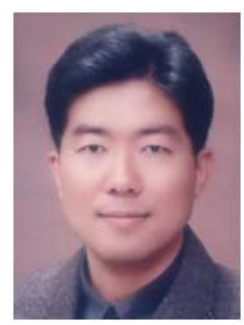

Hyoungsoo Lim received the B.S., M.S., and Ph.D. degrees in electrical engineering from Pohang University of Science and Technology (POSTECH), Pohang, Korea in 1992, 1994, and 1999, respectively. He was with Radio \& Broadcasting Technology Laboratory, Electronics and Telecommunication Research Institute (ETRI), Daejeon, Korea from 1999 to 2000, and DXO Telecom, Inc., Seoul, Korea from 2000 to 2001. He has been with ETRI since 2002, where he is currently a director of Mobile Broadcasting Research Section, Broadcasting System Research Department, ETRI. In 2011, he also joined the faculty of the University of Science \& Technology (UST), Daejeon, Korea as an adjunct professor. His major research interests include Faster-than-Nyquist technologies, OFDM, CDMA, digital broadcasting and communications, military communications, satellite communications, and wireless LAN/MAN/PAN systems. 


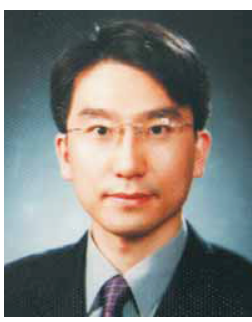

Jaewoo So received the B.S. degree in electronic engineering from Yonsei University, Seoul, Korea, in 1997, and received the M.S. and Ph.D. degrees in electrical engineering from the Korea Advanced Institute of Science and Technology (KAIST), Daejeon, Korea, in 1999 and 2002, respectively. From June 2001 to August 2005, he was with IP One, Seoul, Korea, where he led several research projects and developed IEEE $802.11 \mathrm{a} / \mathrm{b} / \mathrm{g}$ products and heterogeneous network solutions. From September 2005 to December 2007, he was a senior engineer at Samsung Electronics, Suwon, Korea, where he involved in the design, performance evaluation, and development of mobile WiMAX systems and 4G wireless systems. From December 2007 to August 2008, he was a postdoctoral fellow in the Department of Electrical Engineering, Stanford University, Stanford, CA, USA. Since September 2008, he has been with the Department of Electronic Engineering, Sogang University, Seoul, Korea, where he is currently an associate professor. His current research interests include radio resource management, cross-layer design, optimization, protocols, and performance analysis of various communication networks such as cellular systems, wireless networks, cognitive radio networks, and heterogeneous networks. He is a Senior Member of IEEE, a Member of IEICE, a Life Member of KICS, and a Member of IEEK. 